\section{AB1172 NEUROPATHIC PAIN SCREENING TOOLS IN RHEUMATOID ARTHRITIS: REAL WORLD DATA}

T. Martins Rocha ${ }^{1}$, S. Pimenta ${ }^{1}$, M. Bernardes ${ }^{1}$, A. Bernardo ${ }^{1}$, M. Barbosa ${ }^{2}$, R. Lucas ${ }^{3}$, L. Costa ${ }^{1} .{ }^{1}$ Rheumatology; ${ }^{2}$ Pain Unit, Centro Hospitalar de São João; ${ }^{3}$ Clinical Epidemiology, Faculty of Medicine University of Porto (FMUP), Porto, Portugal

Background: The Leeds Assessment of Neuropathic Symptoms (LANSS) and the painDETECT questionnaire (PDQ) are two validated screening tools for neuropathic pain (NP). Recent evidence reported a low level of agreement between these tests in knee Osteoarthritis. Several studies have recently applied the PDQ in Rheumatoid Arthritis (RA), suggesting a NP component in these patients, although the application and performance comparison with LANSS is yet to be studied.

Objectives: Evaluate PDQ and LANSS performance for NP classification and investigate its optimal cutoff points in a RA cohort.

Methods: Observational, cross-sectional study was designed including RA patients followed at our Rheumatology department. Patients with diagnosed neuropathy or non-RA risk factors for NP were excluded. Selected patients were evaluated in a medical visit where LANSS and PDQ were applied. Agreement between the two questionnaires was evaluated using kappa coefficient analysis. Receiver operating characteristic (ROC) analysis was performed using each tool as gold-standard and cutoff points to optimize agreement were investigated. Nonconcordant patients were compared with concordant patients using parametric and non-parametric tests. Significance level was set as $<0.05$.

Results: 112 RA patients were included, $86(77 \%)$ were females, with a mean (SD) age of 55.1 (10.8) years and median disease duration of 13 years (range: 2-41). $102(91 \%)$ were treated with DMARDs and $42 \%$ with a biologic DMARD. $45(40 \%)$ patients had NP applying the LANSS $(\geq 12)$ and $28 \%$ had NP in the PDQ (19 possible and 12 likely; no demographic or clinical significant differences were found between these two groups). 82 (73\%) patients had concordant NP classification (59 negative, 23 positive) by the two tests. Concordant group had significantly superior median disease duration and inferior LANSS scores compared to non-concordant group (14 vs 12 years and 8 vs 13 , respectively, $\mathrm{p}<0.05)$ with no other significant differences found. A moderate agreement $(\kappa=0.41)$ and linear correlation $(r=0.58, p<0.001)$ were observed between the two tests. In the ROC curve analysis, PDQ $(\geq 13)$ showed an area under the curve (AUC) of $0.80,95 \% \mathrm{Cl}[0.72-0.88]$ with a sensitivity and specificity of $51 \%$ and $88 \%$, respectively, using LANSS as gold standard. LANSS $(\geq 12)$ had an AUC of $0.80,95 \% \mathrm{Cl}[0.71-0.90]$ and a sensitivity and specificity of $74 \%$ and $73 \%$, respectively, using PDQ as gold standard. After ROC curve analysis, optimal cutoff for PDQ was 10, showing greater sensitivity (69\%) but lower specificity $(79 \%)$ with a slight increase in the agreement between the tests $(\kappa=0.48)$. For the LANSS, the optimal cutoffs were the previous value or 13 (sensitivity $68 \%$ and specificity $78 \%$ ) with a modest gain in the agreement $(\kappa=0.42)$. Correction for both cutoff points resulted in a more substantial increase in agreement level $(\kappa=0.51)$. Conclusions: In this study, LANSS and PDQ had a moderate level of agreement, possibly because they capture different dimensions of NP. New possible cutoffs were studied to increase agreement between the tests. Further studies with other conditions and a validated gold-standard for NP are needed to confirm this data. References:

[1] Cohen J. Educ Psychol Meas. 1960;20:37-46.

[2] Moreton B.J. et al Arthritis Care Res. 2015 Apr;67(4):519-28.

[3] AW Christensen et al. Scand J Rheumatol 2016;1-9.

Disclosure of Interest: T. Martins Rocha Grant/research support from: Portuguese Society of Rheumatology/Alfa Wassermann on May 2015, S. Pimenta: None declared, M. Bernardes: None declared, A. Bernardo: None declared, M. Barbosa: None declared, R. Lucas: None declared, L. Costa: None declared DOI: 10.1136/annrheumdis-2017-eular.5127

\section{AB1173 PHYSICIAN VISUAL ANALOG SCALE ESTIMATES FOR OVERALL GLOBAL ASSESSMENT, INFLAMMATION, DAMAGE, AND DISTRESS TO ASSESS PATIENTS AND SUPPORT CLINICAL DECISIONS IN ROUTINE RHEUMATOLOGY CARE: ANALYSIS OF INTER-RATER RELIABILITY}

T. Pincus, I. Castrejon, J. Chua, A. Kugasia, J. Schmukler, S. Weinberg, J.A. Block. Rheumatology, Rush University Medical Center, Chicago, United States

Background: A physician global estimate of patient status (DOCGL) was developed to quantify inflammatory activity in rheumatoid arthritis (RA) clinical trials. However, DOCGL may be affected by joint damage and/or distress (in fibromyalgia, depression, etc). One approach to document the possible impact of these problems on DOCGL is to add 3 physician visual analog subscale (VAS) estimates for inflammation, damage, and distress. These subscales have been shown to be useful in patients with diagnoses other than RA (1) but inter-rater reliability has not been analyzed.

Objectives: To analyze inter-rater reliability between senior rheumatologists and trainees on 4 VAS estimates for overall DOCGL, inflammation (DOCINF), damage (DOCDAM) and distress (DOCSTR), in patients with various rheumatic diagnoses. Methods: Patients seen in routine care were assigned 4 physician VAS estimates for overall DOCGL, and levels of inflammation or reversible symptoms (DOCINF), organ damage or irreversible symptoms (DOCDAM), and distress or symptoms not explained by inflammation or damage (DOCSTR). VAS estimates were assigned independently by a senior rheumatologist and a rheumatology trainee for the same patient at the same visit. Mean differences, correlations, and possible discordance of $\geq 2$ units/10 between estimates of the senior rheumatologist and the trainee were analyzed.

Results: VAS estimates by the 2 physicians were analyzed in 64 patients with different rheumatic diseases, including osteoarthritis (16\%), RA (14\%), fibromyalgia (14\%), and systemic lupus erythematosus (13\%). Mean differences of scores assigned by the senior rheumatologists versus trainees were $<0.43 / 10$, less than $5 \%$ of the total scales, slightly lower for DOCINF, and slightly higher for the 3 other subscales $(p<0.001)$ (Table). Mean estimates of both physicians for damage and distress were higher than for inflammation by 1.1 to 1.6 units (Table). Correlations of all 4 VAS between rheumatologists and trainees were significant $(p<0.001)$ (Table). More than $70 \%$ of the estimates were concordant for DOCGL $(75 \%)$, DOCINF $(78 \%)$, and DOCDAM $(70 \%)$, while concordance was somewhat lower for DOCSTR (57\%) (Table).

Conclusions: Good inter-rater agreement between two physicians is seen for 4 VAS estimates for overall global assessment, inflammation, damage, and distress. Mean scores for damage and distress were higher than for inflammation, indicating the complexity of rheumatology care. Quantitative scores can add to documentation of patient status and to support of clinical decisions for doctors, patients, and payers.

\section{References:}

[1] Bull Hosp Jt Dis (2013). 2015 Jul;73(3):178-84.

Disclosure of Interest: T. Pincus Shareholder of: Health Report Services, Inc, I. Castrejon: None declared, J. Chua: None declared, A. Kugasia: None declared, J. Schmukler: None declared, S. Weinberg: None declared, J. Block: None declared DOI: 10.1136/annrheumdis-2017-eular.3526

\section{AB1174 ORM2 AND APOA2 SERUM LEVELS CAN PREDICT OA PATIENT RESPONSE TO CHONDROITIN SULFATE/GLUCOSAMINE HYDROCHLORIDE: RESULTS FROM THE MOVES STUDY}

V. Calamia ${ }^{1}$, M. Camacho-Encina ${ }^{1}$, L. González-Rodríguez ${ }^{1}$

P. Fernández-Puente ${ }^{1}$, I. Rego-Pérez ${ }^{2}$, M. Herrero $^{3}$, H. Martínez $^{3}$,

C. Ruiz-Romero ${ }^{1}$, F.J. Blanco ${ }^{1} .{ }^{1}$ Grupo de

Proteomica-PBR2-ProteoRed/ISCIII-Servicio de Reumatología; ${ }^{2}$ Grupo de

Genómica-Servicio de Reumatología., Instituto de Investigación Biomédica de A Coruña (INIBIC), Complexo Hospitalario Universitario de A Coruña (CHUAC),

Sergas. Universidade da Coruña (UDC)., la Coruña; ${ }^{3}$ Clinical R\&D Area,

Bioiberica S.A., Barcelona, Spain

Background: A shotgun proteomic analysis performed on sera from patients enrolled in the Multicentre Osteoarthritis interVEntion trial with Sysadoa (MOVES) led to the discovery of a panel of putative predictive protein biomarkers useful to stratify osteoarthritis $(\mathrm{OA})$ patients into responders and non-responders, either to Chondroitin sulfate/Glucosamine hydrochloride (Droglican ${ }^{\circledR}$, Bioiberica S.A.,Barcelona,Spain) or Celecoxib.

Objectives: To validate the sensitivity and specificity of a panel of six serum proteins useful to predict the patient response to Droglican treatment, in order to optimize therapeutic outcomes in OA.

Methods: We analyzed the serum levels of a panel of six putative predictive protein biomarkers by enzyme-linked immunosorbent assays (ELISAs): APOA2, APOA4, APOH, C4BPa, ITIH1 and ORM2. All the subjects studied belonged to the MOVES cohort at baseline (Droglican sub-cohort, $n=260$ ). Non-parametric and multivariate analysis were performed to test the effects of the clinical variables, including gender, age, BMI, radiologic Kellgren/Lawrence (K/L) grade and WOMAC score at baseline, as well as the serum levels of each of the six mentioned proteins, on the response to Droglican treatment according to the OMERACT-OARSI criteria and the WOMAC pain score $(20 \%, 30 \%, 50 \%$ and $70 \%$ reduction) recorded at the end of the trial (after 6 months of treatment).

Results: Non parametric analysis showed decreased serum levels of ORM2

Abstract AB1173 - Table 1. Mean and SD for the four physician estimates according to the rheumatologist (rheum) and the trainee, inter-rater reliability and levels of concordance and discordance for each estimate

\begin{tabular}{|c|c|c|c|c|c|c|c|}
\hline \multirow[t]{2}{*}{ VAS $(0-10)$} & \multirow[t]{2}{*}{ Rheum } & \multirow[t]{2}{*}{ Trainee } & \multirow[t]{2}{*}{ Mean Difference } & \multirow[t]{2}{*}{$\begin{array}{l}\text { Pearson } r \\
\text { all } p<0.001\end{array}$} & \multicolumn{3}{|c|}{$\begin{array}{l}\text { Rheumatologist (Rheum) and trainee discordance groups } \\
\text { by } 2 / 10 \text { units, no. (\%) }\end{array}$} \\
\hline & & & & & Rheum $>$ Trainee & Rheum = Trainee & Rheum $<$ Trainee \\
\hline Overall DOCGL & 3.9 (1.9) & $4.0(2.2)$ & $-0.05(1.9)$ & 0.61 & $11(17 \%)$ & $48(75 \%)$ & $5(8 \%)$ \\
\hline DOCINF & $1.7(1.6)$ & $1.4(1.6)$ & $0.28(1.6)$ & 0.50 & $8(13 \%)$ & 50 (78\%) & $6(9 \%)$ \\
\hline DOCDAM & $2.8(2.2)$ & $2.7(2.2)$ & $0.01(2.0)$ & 0.61 & $11(17 \%)$ & 45 (70\%) & $8(12 \%)$ \\
\hline DOCSTR & $3.3(2.9)$ & $2.9(2.4)$ & $0.43(2.8)$ & 0.47 & $12(19 \%)$ & $36(57 \%)$ & $15(24 \%)$ \\
\hline
\end{tabular}

